\title{
Heavy-quark probes of the quark-gluon plasma and interpretation of recent data taken at the BNL Relativistic Heavy Ion Collider
}

\author{
Hendrik van Hees, ${ }^{1}$ Vincenzo Greco, ${ }^{2}$ and Ralf Rapp ${ }^{1}$ \\ ${ }^{1}$ Cyclotron Institute and Physics Department, Texas A\&M University, College Station, Texas 77843-3366, USA \\ ${ }^{2}$ Laboratori Nazionali del Sud INFN, via S. Sofia 62, I-95123 Catania, Italy
}

(Received 31 August 2005; published 29 March 2006)

\begin{abstract}
Thermalization and collective flow of charm $(c)$ and bottom $(b)$ quarks in ultrarelativistic heavy-ion collisions are evaluated based on elastic parton rescattering in an expanding quark-gluon plasma (QGP). We show that resonant interactions in a strongly interacting QGP (sQGP), as well as parton coalescence, can play an essential role in the interpretation of recent data from the BNL Relativistic Heavy-Ion Collider (RHIC), and thus illuminate the nature of the SQGP and its hadronization. Our main assumption, motivated by recent findings in lattice quantum chromodynamics, is the existence of $D$ - and $B$-meson states in the sQGP, providing resonant cross sections for heavy quarks. Pertinent drag and diffusion coefficients are implemented into a relativistic Langevin simulation to compute transverse-momentum spectra and azimuthal asymmetries $\left(v_{2}\right)$ of $b$ - and $c$-quarks in Au-Au collisions at RHIC. After hadronization into $D$ - and $B$-mesons using quark coalescence and fragmentation, associated electron-decay spectra and $v_{2}$ are compared to recent RHIC data. Our results suggest a reevaluation of radiative and elastic quark energy-loss mechanisms in the sQGP.
\end{abstract}

DOI: 10.1103/PhysRevC.73.034913

PACS number(s): $12.38 . \mathrm{Mh}, 24.85 .+\mathrm{p}, 25.75 . \mathrm{Nq}$

\section{INTRODUCTION}

Recent experimental findings at the BNL Relativistic Heavy-Ion Collider (RHIC) have given intriguing evidence for the production of matter at unprecedented (energy-) densities with surprisingly large collectivity and opacity, as reflected by (approximately) hydrodynamic behavior at low transverse momentum $\left(p_{T}\right)$ and a substantial suppression of particles with high $p_{T}$. This has led to the notion of a "strongly interacting Quark-Gluon Plasma" (sQGP), whose microscopic properties, however, remain under intense debate thus far.

Heavy quarks (HQs) are particularly valuable probes of the medium created in heavy-ion reactions, as one expects their production to be restricted to the primordial stages. Recent calculations of radiative gluon energy-loss of charm $(c)$ quarks traversing a QGP in central $\mathrm{Au}-\mathrm{Au}$ collisions at RHIC have found nuclear suppression factors $R_{A A} \simeq 0.3-0.4$ [1,2], comparable to the observed suppression of light hadrons at high $p_{T}$, and in line with preliminary (nonphotonic) single-electron $\left(e^{ \pm}\right)$ decay spectra [3-5]. The latter also exhibit a surprisingly large azimuthal asymmetry $\left(v_{2}\right)[6-8]$ in semicentral Au-Au which cannot be reconciled with radiative energy loss, especially if $c$-quarks are hadronized into $D$-mesons via fragmentation. While the underlying transport coefficients [2] exceed their predicted values from perturbative quantum chromodynamics (pQCD) by at least a factor of $\sim 5$ [9], energy loss due to elastic scattering parametrically dominates toward low $p_{T}$ (by a factor $1 / \sqrt{\alpha_{s}}$ [10]). But elastic pQCD cross sections [11,12] also have to be upscaled substantially to obtain $c$-quark $v_{2}$ and $R_{A A}$ reminiscent to preliminary $e^{ \pm}$data, as shown in a recent Langevin simulation for RHIC [10]. In addition, contributions of bottom $(b)$ quarks [13,14] will reduce the effects in the electron- $R_{A A}$ and $-v_{2}$ at high $p_{T}$. Quark coalescence approaches suggest that an $e^{ \pm}-v_{2}$ in excess of $10 \%$ can only be obtained if [15] (i) light quarks impart their $v_{2}$ on $D$-mesons (see also Refs. [16,17]), (ii) the $c$-quark $v_{2}$ is comparable to that of light quarks.

We are thus confronted with marked discrepancies between pQCD energy-loss calculations and semileptonic heavy-quark (HQ) observables at RHIC. The resolution of this issue is central to the understanding of HQ interactions in the QGP in particular, and to the interpretation of energy loss in general. HQ rescattering also has direct impact on other key observables such as heavy quarkonium production (facilitating regeneration) and dilepton spectra (where $c \bar{c}$ decays compete with thermal QGP radiation).

In this paper we investigate the HQ energy-loss problem by introducing resonant $\mathrm{HQ}$ interactions into a Langevin simulation of an expanding QGP. Our calculations implement a combined coalescence + fragmentation approach for hadronization, as well as bottom contributions, to allow for a quantitative evaluation of pertinent $e^{ \pm}$-spectra $\left(v_{2}\right.$ and $\left.R_{A A}\right)$ which is mandatory for a proper interpretation of recent RHIC data. Our main assumption of resonant $D$ - and $B$-like states in the sQGP has been shown [18] to reduce HQ thermalization times by a factor of $\sim 3$ compared to $\mathrm{pQCD}$ scattering. Theoretical evidence for resonances in the sQGP derives from computations of heavy and light meson correlators within lattice QCD (1QCD) [19,20], as well as applications of lQCDbased heavy-quark potentials within effective models [21-24]. Except for the mass and width of these states, no further free parameters enter our description, with degeneracies based on chiral and HQ symmetry.

\section{HEAVY-QUARK INTERACTIONS IN THE QGP}

Following Ref. [18] our description of HQ interactions in the QGP focuses on elastic scattering, mediated by resonance excitations on light antiquarks $(\bar{q})$ as well as (nonresonant) 
leading order pQCD processes dominated by $t$-channel gluon exchange. The latter correspond to Born diagrams [25] regularized by a gluon-screening mass $m_{g}=g T$ with a strong coupling constant, $\alpha_{s}=g^{2} /(4 \pi)=0.4$. The key assumption [18] is that a QGP at moderate temperatures $T \leqslant 2 T_{c}$ sustains strong correlations in the lowest-lying color-neutral $D$ - and $B$-meson channels. Support for the relevance of such interactions stems from quenched 1QCD computations of euclidean mesonic correlation functions, which, after transformation into the timelike regime, exhibit resonance structures for both (heavy) $Q-\bar{Q}$ and (light) $q-\bar{q}$ states $[19,20]$. In addition, applications of lQCD-based $Q-\bar{Q}$ potentials have revealed both bound [21,22] and resonance states [23] with dissolution temperatures of $\sim 2 T_{c}$, quite compatible with the disappearance of the peak structures in the 1QCD spectral functions. Here, we do not attempt a microscopic description of these correlations but cast them into an effective lagrangian with $\bar{q}-Q-\Phi$ vertices $(\Phi=$ $D, B)$, at the price of two free parameters: the masses of the meson-fields, fixed at $m_{D(B)}=2(5) \mathrm{GeV}$, i.e., $0.5 \mathrm{GeV}$ above the $Q-\bar{q}$ threshold (with quark masses $m_{c(b)}=1.5(4.5) \mathrm{GeV}$, $\left.m_{u, d}=0\right)$, and their width, $\Gamma$, obtained from the one-loop $\Phi$ self-energy (which, in turn, dresses the $\Phi$-propagator) with the pertinent coupling constant varied to cover a range suggested by effective quark models [26,27], $\Gamma=0.4-0.75 \mathrm{GeV}$. The multiplicity of $\Phi$ states follows from chiral and HQ symmetries alone, implying degenerate $J^{P}=0^{ \pm}$and $1^{ \pm}$states. We emphasize again that, besides the mass and width of the $\Phi$ states, no other free parameters (or scale factors) are introduced.

The matrix elements for resonant and pQCD scattering are employed to calculate drag and diffusion coefficients of HQs in a Fokker-Planck approach [11]. Resonances reduce the thermalization times for both $c$ - and $b$-quarks by a factor of $\sim 3$ compared to pQCD scattering alone [18].

\section{LANGEVIN SIMULATION}

To evaluate thermalization and collective flow of HQs in $\mathrm{Au}-\mathrm{Au}$ collisions we perform relativistic Langevin simulations [10] embedded into an expanding QGP fireball. In the local rest frame of the bulk matter, the change in position $(\vec{x})$ and momentum $(\vec{p})$ of $c$ - and $b$-quarks during a time step $\delta t$ is defined by

$$
\delta \vec{x}=\frac{\vec{p}}{E} \delta t, \quad \delta \vec{p}=-A(t, \vec{p}+\delta \vec{p}) \vec{p} \delta t+\delta \vec{W}(t, \vec{p}+\delta \vec{p})
$$

( $E$ : HQ energy), where $\delta \vec{W}$ represents a random force which is distributed according to Gaussian noise [34],

$$
P(\delta \vec{W}) \propto \exp \left[-\frac{\hat{B}_{j k} \delta W^{j} \delta W^{k}}{4 \delta t}\right] .
$$

The drag coefficient (inverse relaxation time), $A$, and the inverse of the diffusion-coefficient matrix,

$$
B_{j k}=B_{0}\left(\delta^{j k}-\hat{p}^{j} \hat{p}^{k}\right)+B_{1} \hat{p}^{j} \hat{p}^{k},
$$

are given by the microscopic model of Ref. [18], including $p$ and $T$-dependencies (the latter converts into a time dependence using the fireball model described below). The longitudinal diffusion coefficient is set in accordance with Einstein's dissipation-fluctuation relation to $[10] B_{1}=T E A$, to ensure the proper thermal equilibrium limit. The latter also requires care in the realization of the stochastic process in Eq. (1); we here use the so-called Hänggi-Klimontovich realization [34] which approaches a relativistic Maxwell distribution if the Einstein relation is satisfied (in the Ito realization, e.g., an extra term has to be introduced in $A$ [35]). Finally, the HQ momenta are Lorentz-boosted to the laboratory frame with the velocity of the bulk matter at the actual position of the quark, as determined by the fireball flow profile (see below).

The time evolution of Au-Au collisions is modeled by an isentropically expanding, isotropic QGP fireball with a total entropy fixed to reproduce measured particle multiplicities at hadro-chemical freezeout which we assume to coincide with the phase transition at $T_{c}=180 \mathrm{MeV}$ [28]. The temperature at each instant of time is extracted from an ideal QGP equation of state with an effective flavor degeneracy of $N_{f}=2.5$. Radial and elliptic flow of the bulk matter are parameterized to closely resemble the time-dependence found in hydrodynamical calculations [29], assuming a flow profile rising linearly with the radius. We focus on semicentral collisions at impact parameter $b=7 \mathrm{fm}$, with an initial spatial eccentricity of 0.6 and a formation time of $\tau_{0}=0.33 \mathrm{fm} / c$, translating into an initial temperature of $T_{0}=340 \mathrm{MeV}$. The evolution is terminated at the end of the QGP-hadron gas mixed phase (constructed via standard entropy balance [28]) after about $5 \mathrm{fm} / c$, at which point the surface flow velocity and momentum anisotropy have reached $v_{\perp}=0.5 \mathrm{c}$ and $v_{2}=5.5 \%$ (variations in $\tau_{0}$ by a factor of two affect the $c$-quark $v_{2}$ and $R_{A A}$ by $10-20 \%$ (less for the $e^{ \pm}$spectra), while a reduction in the critical temperature to $170 \mathrm{MeV}$ increases (decreases) $v_{2}^{c}\left(R_{A A}\right)$ somewhat less. $D$-meson rescattering in the hadronic phase [30] is neglected).

To specify initial HQ $p_{T}$-distributions, $P_{\text {ini }}\left(p_{T}\right)$, and especially the relative magnitude of $c$ - and $b$-quark spectra (essential for the evaluation of $e^{ \pm}$spectra), we proceed as follows: we first use modified PYTHIA $c$-quark spectra with $\delta$-function fragmentation to fit $D$ and $D^{*}$ spectra in $\mathrm{d}$-Au collisions [31]. These spectra are decayed to single- $e^{ \pm}$ which saturate pertinent data from $p-p$ and $\mathrm{d}-\mathrm{Au}$ up to $p_{T}^{e} \simeq 3.5 \mathrm{GeV}[32,33]$. The missing yield at higher $p_{T}$ is attributed to contributions from $B$-mesons, resulting in a cross section ratio of $\sigma_{b \bar{b}} / \sigma_{c \bar{c}} \simeq 5 \cdot 10^{-3}$ (which is slightly smaller than for pQCD predictions [13] and implies a crossing of $c$ and $b$-decay electrons at $p_{T} \simeq 5 \mathrm{GeV}$, as compared to $4 \mathrm{GeV}$ in $\mathrm{pQCD}$ ).

Figure 1 summarizes the output of the Langevin simulations for the HQ nuclear modification factor, $R_{A A}=$ $P_{\text {fin }}\left(p_{T}\right) / P_{\text {ini }}\left(p_{T}\right)$ ( $P_{\text {fin }}$ : final $p_{T}$-distributions $)$, and elliptic flow, $v_{2}=\left\langle\left(p_{x}^{2}-p_{y}^{2}\right) /\left(p_{x}^{2}+p_{y}^{2}\right)\right\rangle_{p_{T}}$ (evaluated at fixed $\left.p_{T}\right)$. For $c$-quarks and pQCD scattering only, our results are in fair agreement with those of Ref. [10] (recall that the Debye mass in our calculations is given by $m_{g}=g T$ while in Ref. [10] it was set to $m_{g}=1.5 T$ independent of $\alpha_{s}$ ). However, both $R_{A A}$ and $v_{2}$ exhibit substantial sensitivity to the inclusion of resonance contributions, increasing the effects of pQCD scattering by a factor of $\sim 3-5$. Also note the development of the plateau in $v_{2}\left(p_{T}>3 \mathrm{GeV}\right)$ characteristic for incomplete thermalization of HQs in the bulk matter. 

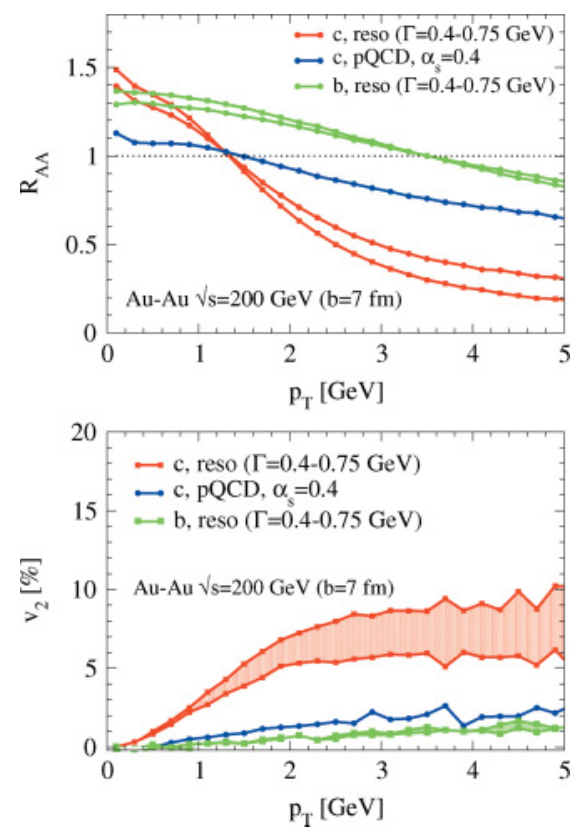

FIG. 1. (Color online) Nuclear modification factor (upper panel) and elliptic flow (lower panel) of charm and bottom quarks in $b=$ $7 \mathrm{fm} \mathrm{Au}-\mathrm{Au}(\sqrt{s}=200 \mathrm{GeV})$ collisions based on elastic rescattering in the QGP. Red (green) and blue lines are for $c$ - $(b$-) quarks with and without resonance rescattering, respectively, where the bands encompass resonance widths of $\Gamma=0.4-0.75 \mathrm{GeV}$.

\section{HADRONIZATION AND SINGLE-ELECTRON SPECTRA}

Semileptonic single- $e^{ \pm}$spectra are a valuable tool to investigate heavy-meson spectra in ultrarelativistic heavy-ion collisions, since their decay kinematics largely conserves the spectral properties of the parent particles $[15,36]$. To compare our results to measured single- $e^{ \pm}$in Au-Au collisions, the above HQ spectra have to be hadronized. To this end we employ the coalescence approach of Ref. [15] based on earlier constructed light-quark spectra [37]. Quark coalescence has recently enjoyed considerable success in describing, e.g., the "partonic scaling" of elliptic flow and the large $p / \pi$ ratio in $\mathrm{Au}-\mathrm{Au}$ at RHIC [37-39], as well as flavor asymmetries in $D$-meson production in elementary hadronic collisions [40]. Whereas at low $p_{T}$ most of the HQs coalesce into $D$ - and $B$-mesons, this is no longer the case at higher $p_{T}$ where the phase space density of light quarks rapidly decreases. Therefore, to conserve HQ number in the $B$ and $D$-meson spectra, the remaining $c$ - and $b$-quarks are hadronized using $\delta$-function fragmentation. Finally, single- $e^{ \pm}$ $p_{T^{-}}$and $v_{2}$-spectra are computed via $B$ - and $D$-meson 3-body decays, and compared to experiment in Fig. 2. We find that the effects of resonances are essential in improving the agreement with data, both in terms of lowering the $R_{A A}$ and increasing $v_{2}$. The $B$-meson contribution reflects itself by limiting $R_{A A}$ and $v_{2}$ to values above 0.4 and below $10 \%$, respectively, as well as the reduction of $v_{2}$ above $p_{T} \simeq$ $3 \mathrm{GeV}$.

To better illustrate the effects of coalescence we plot in Fig. 3 calculations where all HQs are fragmented into $D$ - and
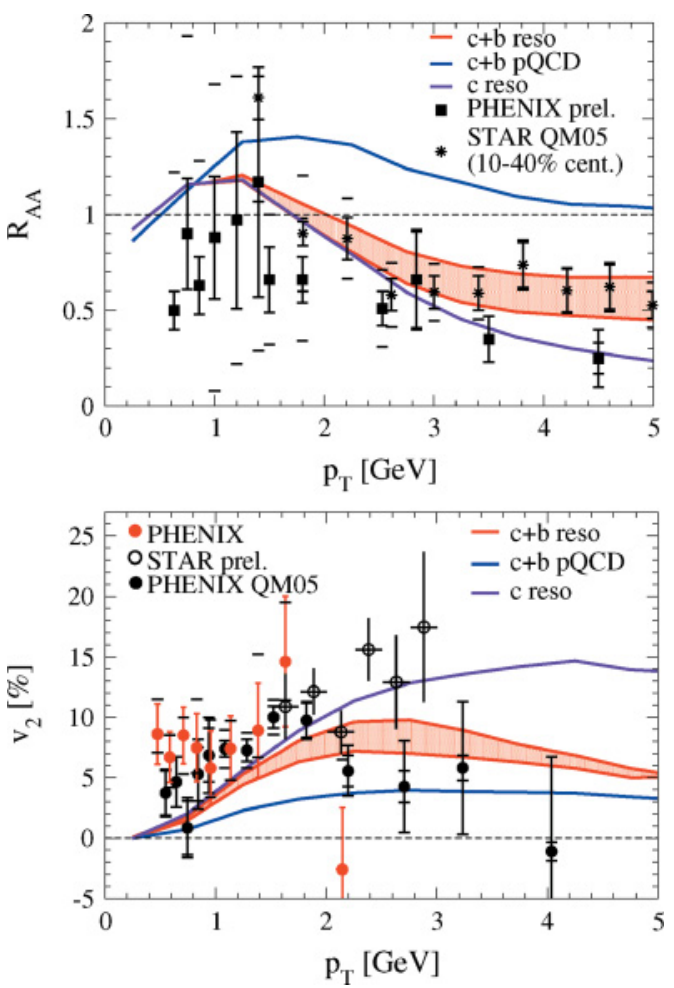

FIG. 2. (Color online) $R_{A A}$ (upper panel) and $v_{2}$ (lower panel) of semileptonic $D$ - and $B$-meson decay-electrons in $b=7 \mathrm{fm}$ $\mathrm{Au}-\mathrm{Au}(\sqrt{s}=200 \mathrm{GeV})$ using HQ coalescence supplemented by fragmentation (line identification as in Fig 1). The data [4,6-8] are for minimum bias collisions, the $R_{A A}$ from STAR [5] for $10-40 \%$ central collisions.
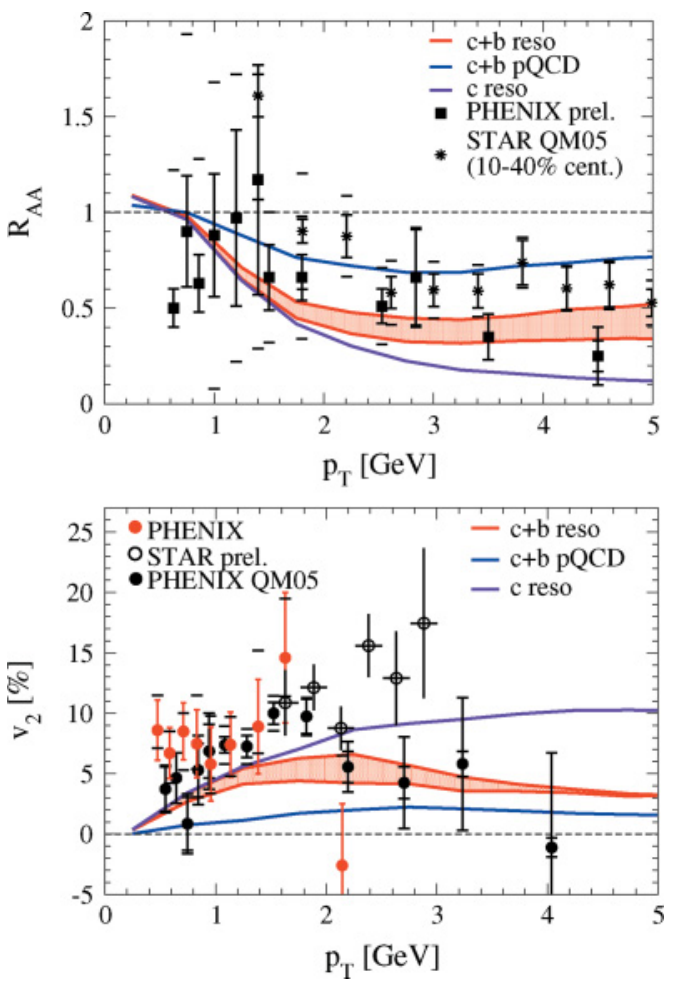

FIG. 3. (Color online) Same as Fig. 2 but using HQ fragmentation only. 
$B$-mesons. While $R_{A A}$ is significantly reduced, most notably in the $p_{T} \simeq 1-2 \mathrm{GeV}$ region, $v_{2}$ also decreases reaching at most $6 \%$, which is not favored by current data. It is, however, conceivable that modifications in the fraction of coalescence to fragmentation contributions, as well as improvements in our schematic ( $\delta$-function) treatment of fragmentation, will be necessary once more accurate experimental data become available. Additional corrections may also arise from a more precise determination of the $b / c$ ratio and nuclear shadowing.

\section{CONCLUSION}

We have investigated thermalization and collective flow of $c$ - and $b$-quarks within a relativistic Langevin approach employing elastic scattering in an expanding QGP fireball in semicentral $\mathrm{Au}-\mathrm{Au}$ collisions at RHIC. Underlying drag and diffusion coefficients were evaluated assuming resonant $D$ and $B$-meson correlations in the sQGP, enhancing heavy-quark rescattering. Corresponding $p_{T}$-spectra and elliptic flow of $c$-quarks exhibit a large sensitivity to the resonance effects, lowering $R_{A A}$ down to 0.2 and raising $v_{2}$ up to $10 \%$, while the impact on $b$-quarks is small. Heavy-light quark coalescence in subsequent hadronization significantly amplifies the $v_{2}$ in single-electron decay spectra, but also increases their $R_{A A}$, especially in the $p_{T} \simeq 2 \mathrm{GeV}$ region. Bottom contributions dominate above $3.5 \mathrm{GeV}$ reducing both suppression and elliptic flow. The combined effects of coalescence and resonant heavy-quark interactions are essential in generating a $v_{2}^{e}$ of up to $10 \%$, together with $R_{A A}^{e} \simeq 0.5$, supplying a viable explanation of current electron data at RHIC without introducing extra scale factors. Our analysis thus suggests that elastic rescattering of heavy quarks in the sQGP is an important component for the understanding of heavy-flavor and single-electron observables in heavy-ion reactions at collider energies. While induced gluon-radiation is expected to be the prevalent interaction with the medium at sufficiently high $p_{T}[1,2,14]$, it may not be the dominant effect below $p_{T} \simeq 6 \mathrm{GeV}$ or so. A complete picture should clearly include both elastic and inelastic rescattering mechanisms.

\section{ACKNOWLEDGMENTS}

One of us (H.vH.) thanks the Alexander von Humboldt foundation for support within a Feodor Lynen fellowship. This work was supported in part by a U.S. National Science Foundation CAREER award under grant PHY-0449489.
[1] M. Djordjevic, M. Gyulassy, and S. Wicks, Phys. Rev. Lett. 94, 112301 (2005).

[2] N. Armesto, A. Dainese, C. A. Salgado, and U. A. Wiedemann, Phys. Rev. D 71, 054027 (2005).

[3] S. S. Adler et al. (PHENIX Collaboration), Phys. Rev. Lett. 94, 082301 (2005).

[4] B. Jacak et al. (PHENIX Collaboration), nucl-ex/0508036.

[5] J. Bielcik et al. (STAR Collaboration), nucl-ex/0511005.

[6] S. S. Adler et al. (PHENIX Collaboration), Phys. Rev. C 72, 024901 (2005).

[7] F. Laue et al. (STAR Collaboration), J. Phys. G 31, S27 (2005).

[8] Y. Akiba et al. (PHENIX Collaboration), nucl-ex/0510008.

[9] R. Baier, Nucl. Phys. A715, 209 (2003).

[10] G. D. Moore and D. Teaney, Phys. Rev. C 71, 064904 (2005).

[11] B. Svetitsky, Phys. Rev. D 37, 2484 (1988).

[12] M. G. Mustafa and M. H. Thoma, Acta Phys. Hung. A 22, 93 (2005).

[13] M. Cacciari, P. Nason, and R. Vogt, Phys. Rev. Lett. 95, 122001 (2005).

[14] M. Djordjevic, M. Gyulassy, and R. Vogt, Phys. Lett. B632, 81 (2006).

[15] V. Greco, C. M. Ko, and R. Rapp, Phys. Lett. B595, 202 (2004).

[16] D. Molnar, J. Phys. G 31, S421 (2005).

[17] B. Zhang, L. W. Chen, and C. M. Ko, Phys. Rev. C 72, 024906 (2005).

[18] H. van Hees and R. Rapp, Phys. Rev. C 71, 034907 (2005).

[19] M. Asakawa and T. Hatsuda, Phys. Rev. Lett. 92, 012001 (2004); Nucl. Phys. A721, 869 (2003).
[20] F. Karsch and E. Laermann, hep-lat/0305025.

[21] E. V. Shuryak and I. Zahed, Phys. Rev. C 70, 021901(R) (2004).

[22] C. Y. Wong, Phys. Rev. C 72, 034906 (2005).

[23] M. Mannarelli and R. Rapp, Phys. Rev. C 72, 064905 (2005).

[24] W. M. Alberico et al., Phys. Rev. D 72, 114011 (2005).

[25] B. L. Combridge, Nucl. Phys. B151, 429 (1979).

[26] F. O. Gottfried and S. P. Klevansky, Phys. Lett. B286, 221 (1992).

[27] D. Blaschke, G. Burau, Yu. L. Kalinovsky, and V. L. Yudichev, Prog. Theor. Phys. Suppl. 149, 182 (2003).

[28] R. Rapp, Phys. Rev. C 63, 054907 (2001).

[29] P. F. Kolb, J. Sollfrank, and U. Heinz, Phys. Rev. C 62, 054909 (2000).

[30] C. Fuchs, B. V. Martemyanov, A. Fässler, and M. I. Krivoruchenko, Phys. Rev. C 73, 035204 (2006).

[31] J. Adams et al. (STAR Collaboration), Phys. Rev. Lett. 94, 062301 (2005)

[32] S. S. Adler et al. (PHENIX Collaboration), Phys. Rev. Lett. 96, 032001 (2006).

[33] A. Tai et al. (STAR Collaboration), J. Phys. G 30, S809 (2004).

[34] J. Dunkel and P. Hänggi, Phys. Rev. E 71, 016124 (2005).

[35] P. Arnold, Phys. Rev. E 61, 6099 (2000).

[36] X. Dong et al., Phys. Lett. B597, 328 (2004).

[37] V. Greco, C. M. Ko, and P. Levai, Phys. Rev. C 68, 034904 (2003).

[38] R. C. Hwa and C. B. Yang, Phys. Rev. C 67, 034902 (2003).

[39] R. J. Fries, B. Muller, C. Nonaka, and S. A. Bass, Phys. Rev. C 68, 044902 (2003).

[40] R. Rapp and E. V. Shuryak, Phys. Rev. D 67, 074036 (2003). 